\title{
EXPERIMENTAL INVESTIGATION OF THERMOCAPILLARY MIGRATION OF ISOLATED DROPS
}

\author{
J.C. Xie ${ }^{1}$, H. Lin ${ }^{1}$, J. H. Han ${ }^{1}$, X.Q. Dong ${ }^{1}$, W.R. Hu ${ }^{1}$, A. Hirata ${ }^{2}$ and M. Sakurai ${ }^{2}$ \\ ${ }^{1}$ Institute of Mechanics, Chinese Academy of Sciences, Beijing 100080, China \\ ${ }^{2}$ Department of Chemical Engineering, Waseda University, Tokyo 169, Japan
}

\begin{abstract}
Experimental hardware has been developed to perform experiments on the Marangoni migration of drops in the case of intermediate Reynolds numbers in a microgravity environment. The experiments were conducted using the drop shaft free fall facility with a 4.5 second microgravity period in the Microgravity Laboratory of Japan. In this experiment, the thermocapillary velocity of drop migration was measured for drops of different sizes in a series of temperature gradients. (C) 1999 COSPAR. Published by Elsevier Science Ltd.
\end{abstract}

\section{INTRODUCTION}

The migration of drops with a diameter of a few millimeters for intermediate Reynolds number $O\left(10^{1}\right)$ in magnitude has been studied experimentally on the ground (Xie et al., 1996). In this case, the thermocapillary effect is coupled with the gravity effect. The present paper discusses an experiment performed in microgravity by using the free fall facilities of a drop shaft. The aim of the experiment was to study the Marangoni migration of drops driven only by thermocapillarity and making a comparison with the prediction of linear theory.

Marangoni migration of a single drop or bubble was first studied by Young, Goldstein and Block as YGB model (1959). It has extensive applications in the field of chemical engineering and processes of heat and mass transfer. The gravity effect and thermocapillary effect are usually coupled in the ground-based process. The subject of Marangoni drop migration becomes more attractive in microgravity science because the interfacial phenomena is important in the microgravity environment where the gravity effect is greatly reduced and the drop migration is dominated by the thermocapillary effect. The linear theory of the YGB model omitted the inertia influence for the case of small Reynolds number and convective energy transport for the case of small Peclet number. The migration velocity of the YGB model may be expressed as follows in the cases wherc the applied temperature gradient is parallel to the direction of gravity

where

$$
\begin{aligned}
& \mathrm{V}=\mathrm{V}_{\mathrm{g}}+\mathrm{V}_{\mathrm{T}}, \\
& \mathrm{V}_{\mathrm{g}}=\mathrm{AR} \mathrm{R}^{2}, \mathrm{~V}_{\mathrm{l}}=\mathrm{B} \Gamma \mathrm{R},
\end{aligned}
$$

$$
A=\frac{2 g\left(\rho-\rho^{\prime}\right)\left(\mu+\mu^{\prime}\right)}{3 \mu\left(2 \mu+3 \mu^{\prime}\right)}, B=\frac{d \sigma}{d T} \frac{2 \kappa}{\left(2 \mu+3 \mu^{\prime}\right)\left(2 \kappa+\kappa^{\prime}\right)},
$$


$\mathrm{R}$ is the radius of the drop. $\sigma, \mu, \rho$, and $\kappa$ are the interfacial tension, the viscosity, the liquid density and the thermal conductivity of continuous phase, respectively. Superscript ' represents the quantities of the drop phase. $\mathrm{d} \sigma / \mathrm{dT}$ and $\Gamma=\mathrm{dT} / \mathrm{dz}$ are respectively the gradient of interfacial tension and vertical temperature gradient at infinite. Analytical solutions were obtained to include the influence of inertia (Balasubramaniam and Cai, 1987; Haj-Hariri et al., 1990), or the influence of convective heat transfer (Subramanian, 1981; Shankar and Subramanian, 1988; Merritt et al., 1993), however, of large Reynolds numbers and large Peclet number can only be solved by the method of numerical simulation (Balasubramaniam and Lavery, 1989).

A few microgravity experiments on the thermocapillary migration of drops have been performed on board the microgravity sounding rocket and spacelab. The results of the small drops with a $11 \pm 1.5 \mu \mathrm{m}$ diameter agree with the theory of the YGB model (Braun et al., 1993), however, the migration velocities of larger drops with a 0.69-2.38 mm diameter are smaller than the ones given by the YGB model (Wozniak, 1991). More experiments have been completed on the ground, and the migration velocity may be obtained with the coupled effects of gravity and thermocapillarity. The coupled effects may be separated only in the case of small drops and a small applied temperature gradient, see for example, (Young et al., 1959; Hahnel et al., 1989; Nallani and Subramanian, 1993). The ground-based experimental results show that the coupled migration velocities of drops are smaller than the ones of linear model (Xie et al., 1996). In the present paper, the microgravity experiment of drop shaft gives the similar results. Both conclusions agree generally with the experiment performed in a sounding rocket (Wozniak, 1991).

\section{EXPERIMENT MEDIUMS}

Immiscible vegetable oil and 5cst silicone oil were used as experiment media of the matrix liquid and drop, respectively, in this experiment. The vegetable oil had the same density as $5 \mathrm{cst}$ silicone oil at about $15^{\circ} \mathrm{C}$. They are immiscible at around room temperature. The majority of the necessary liquid properties were measured for the drop shaft experiment. The measurement errors are within $2 \%$ for $\sigma$, and smaller than $5 \%$ for the other physical parameters. The properties of this liquid system were measured using the same methods as were used for the ground-based experiment (Xie et al., 1996). The major properties of the liquid system used in the present experiment are summarized in Table 1.

Table 1. Physical parameters of liquids.

\begin{tabular}{|c|l|c|c|c|c|}
\hline \multicolumn{2}{|c|}{ Temp. } & $15^{\circ} \mathrm{C}$ & $35^{\circ} \mathrm{C}$ & $50^{\circ} \mathrm{C}$ & $70^{\circ} \mathrm{C}$ \\
\hline \multirow{4}{*}{$\begin{array}{c}\text { Silicone } \\
\text { oil }\end{array}$} & $\mu^{\prime}\left(c p=10^{-2} \mathrm{dyn} \cdot \mathrm{s} / \mathrm{cm}^{2}\right)$ & 5.09 & 3.61 & 2.86 & 2.17 \\
\cline { 2 - 6 } & $\mathrm{K}^{\prime}(\mathrm{mw} / \mathrm{mK})$ & 112 & 111 & 107 & 105 \\
\cline { 2 - 6 } & $\rho^{\prime}\left(\mathrm{g} / \mathrm{cm}^{3}\right)$ & 0.919 & 0.902 & 0.887 & 0.869 \\
\cline { 2 - 6 } & $\beta^{\prime}(1 / \mathrm{K})$ & 0.00118 & 0.00118 & 0.00118 & 0.00118 \\
\hline \multirow{4}{*}{$\begin{array}{c}\text { Vegetable } \\
\text { oil }\end{array}$} & $\mu\left(c p=10^{-2} d y n \cdot s / \mathrm{cm}^{2}\right)$ & 92.2 & 39.7 & 23.7 & 13.67 \\
\cline { 2 - 6 } & $\mathrm{K}(\mathrm{mw} / \mathrm{mK})$ & 176 & 175 & 173 & 171 \\
\cline { 2 - 6 } & $\rho\left(\mathrm{g} / \mathrm{cm}^{3}\right)$ & 0.919 & 0.907 & 0.897 & 0.886 \\
\cline { 2 - 6 } & $\beta(/ \mathrm{K})$ & 0.00081 & 0.00081 & 0.00081 & 0.00081 \\
\hline \multirow{5}{*}{} & $\sigma(d y n / \mathrm{cm})$ & $-0.031^{*}$ & 1.18 & 0.722 & 0.422 \\
\cline { 2 - 6 } & $\frac{d \sigma}{d T}(d y n / \mathrm{cm} \cdot \mathrm{K})$ & -0.0322 & -0.0172 & -0.0152 \\
\hline
\end{tabular}

* The data are given by extrapolation. 


\section{EXPERIMENTAL APPARATUS AND PROCEDURE}

The experimental apparatus of the present experiment is basically the same as the one used in the groundbased experiment (Xie et al., 1996). In the present experiment of drop shaft, the drop capsule is divided into three levels for integrating the experiment facility. Two drop migration systems were packed in the capsule so that two experiments can be performed at the same time during each free fall. The experimental procedure is designed to be automatically controlled by computer system, and the system may also be operated by hand in need. The experimental facility can control the experimental conditions, such as drop size and drop numbers as required by tele-operation. The moving drops were monitored and recorded on-line by a CCD video camera, and drop diameters, drop migration trajectories, and velocities can be obtained by analysis of image data. The matrix liquid was preheated more than one hour to keep the temperature gradient before the free fall and the nearly approximate linear temperature profiles were obtained. The drop shaft facilities in MGLAB offers about 4.5 seconds of microgravity period. This period is short for the drop to migrate to its steady velocity. The migration process for intermediate Reynolds numbers may be observed by injecting a drop just before the start of free fall due to the limited microgravity period, and this procedure was completed in the present experiment. The drop is designed to migrate slowly upward in the test cell, due to the buoyancy and thermocapillary effects before the free fall, and the drop continues to migrate in microgravity environment during the free fall. In this case, the disadvantage of the short microgravity period may be overcome partly because the starting period of moving drop from rest is avoided. Apparently, the migration velocity is much higher under the joint effects of buoyancy and thermocapillarity than that when the drop was driven only by thermocapillary effect in microgravity, because the density of drop liquid is smaller than that of matrix liquid.

\section{EXPERIMENT RESULTS}

The experiment results clearly show the velocity feature before and during the free fall. As an example, Figure 1 gives the history of migration velocity from the normal gravity to microgravity under a temperature gradient $20^{\circ} \mathrm{C} / \mathrm{cm}$. The drop diameter was $5.56 \mathrm{~mm}$ and microgravity started when the drop moved to $27 \mathrm{~mm}$ from the bottom of the test cell. The total height of the test cell is $33 \mathrm{~mm}$. It should be

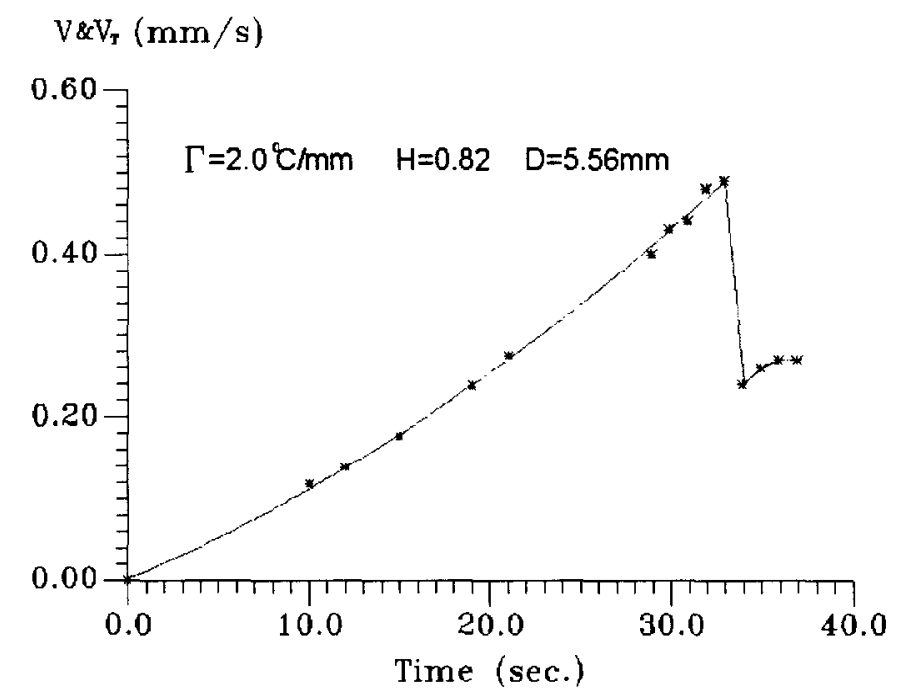

Fig. 1. The typical time history and transition process of drop migration velocity from one-g to micro-g. 
pointed out that, because the vertical temperature gradient was persistent in the test cell, the thermocapillary force always acted on the drop from the time when the drop was injected into the test cell throughout the period of free fall, and buoyancy acted on the drop in addition to the thermocapillary force before the free fall. It can be seen in Figure 1, that the migration velocity was changed at the moment when gravity was altered from one-g to micro-g. The thermocapillary migration velocity in microgravity was much lower than the velocity driven by the combined effects of buoyancy and thermocapillarity. The experiment results reveal the tendency of the migration velocity to gradually increase to a steady value if the migration distance is long enough and the vertical temperature gradient is uniform. The analysis of experimental result show that the observed drop thermocapillary velocities have nearly approached the steady velocity values in the present experiment. Figure 1 indicates the migration velocity variation which decreases a lot when the gravitational level changes from one-g to micro-g. This implies that there must be a resistance which reduces the migration velocity during the short duration when the buoyant force no longer exists.

The comparison of experimental thermocapillary velocities with those of the YGB model under different temperature gradients is shown in figure 2 , respectively. The thermocapillary velocities in microgravity in the present experiment are smaller than those given by the YGB linear model for all the four cases of temperature gradient. Compared to smaller drops, larger drops give larger thermocapillary velocities for fixed temperature gradients. This agrees with the ground-based experiment results (Xie et al., 1996).
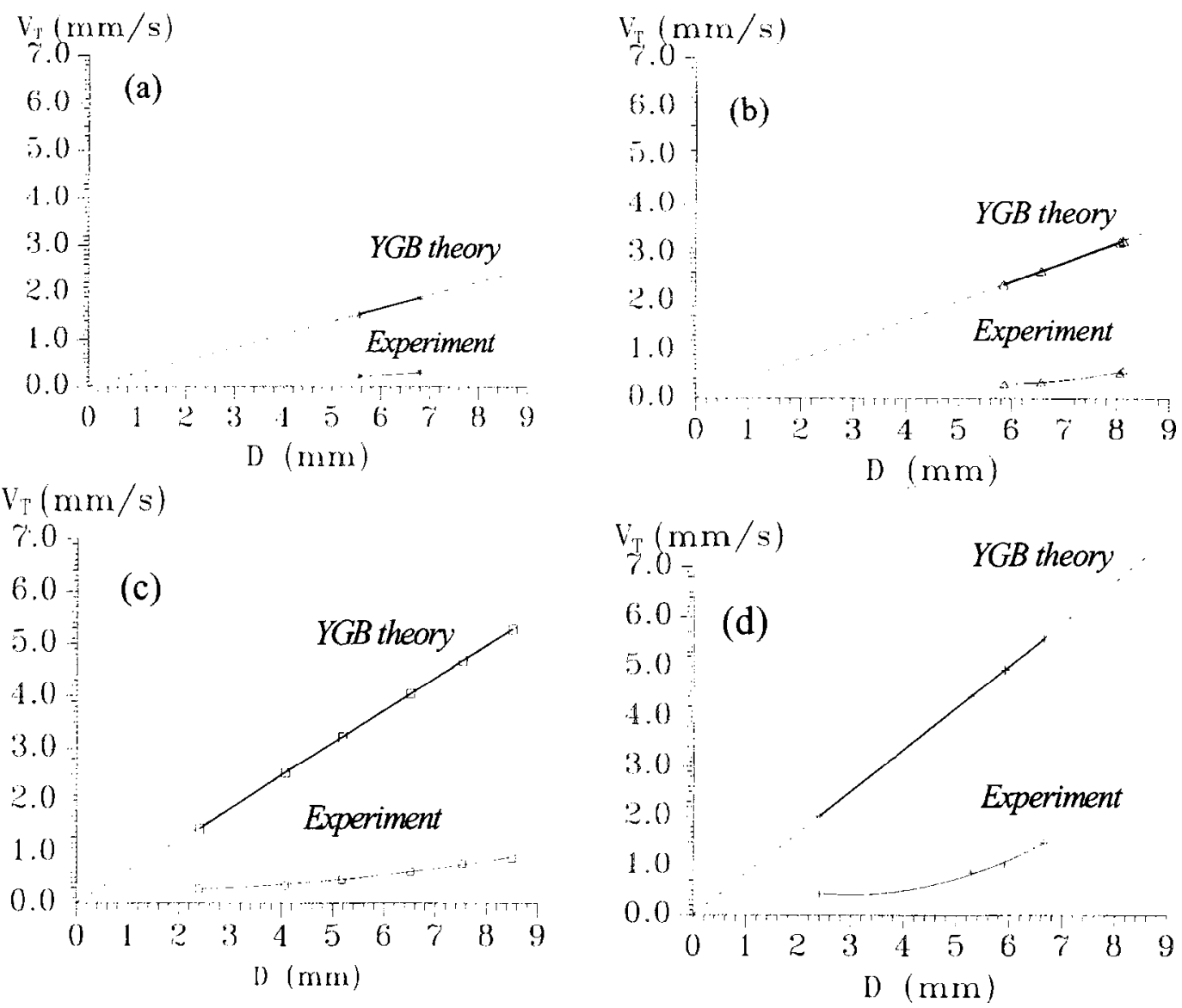

Fig. 2. Comparisons between the experimental data and the YGB linear theory for temperature gradients $\Gamma=2.0^{\circ} \mathrm{C} / \mathrm{mm}(\mathrm{a}), 2.4^{\circ} \mathrm{C} / \mathrm{mm}(\mathrm{b}), 3.2^{\circ} \mathrm{C} / \mathrm{mm}(\mathrm{c})$ and $3.8^{\circ} \mathrm{C} / \mathrm{mm}(\mathrm{d})$, respectively. 
The measured thermocapillary velocities of drop migration and the corresponding Reynolds numbers are reported in Table 2.

Table 2. Results from analysis of the measured data

\begin{tabular}{|c|c|c|c|}
\hline $\begin{array}{c}\text { temperature gradient } \\
\Gamma\left({ }^{0} \mathrm{C} / \mathrm{cm}\right)\end{array}$ & $\begin{array}{c}\text { drop diameter } \\
\mathrm{D}(\mathrm{mm})\end{array}$ & $\begin{array}{c}\text { Thermocapillary velocity } \\
V_{T}(\mathrm{~mm} / \mathrm{s})\end{array}$ & $\begin{array}{c}\text { Reynolds number } \\
\operatorname{Re}\end{array}$ \\
\hline 38 & $2.40-6.65$ & $0.43-1.49$ & $0.44-4.25$ \\
\hline 32 & $2.40-8.50$ & $0.28-0.88$ & $0.25-2.82$ \\
\hline 24 & $5.87-8.13$ & $0.29-0.56$ & $0.57-1.51$ \\
\hline 20 & $5.56-6.80$ & $0.21-0.30$ & $0.45-0.64$ \\
\hline
\end{tabular}

By successfully controlling the drop size in the experiment, the influence of temperature gradient on velocity could be investigated. Figure 3 gives the thermocopillary velocities versus the applied temperature gradients for some related drops. In addition, the corresponding thermocapillary velocities given by the YGB model are also shown in this figure to make a comparison with the experimental data. It can be seen that the thermocapillary velocity is proportional to the applied temperature gradient for a fixed drop diameter, and both the experimental results and the YGB linear theory have the same conclusion, however, according to equation (1.2) and (1.3), the YGB model gives a linear relation of thermocapillary velocity versus temperature gradient, but, the experimental results shows certain nonlinear relations instead. The thermocapillary velocities obtained experimentally are much lower than their counterparts given by the linear theory.

$V_{V} /(\mathrm{mm} / \mathrm{s})$

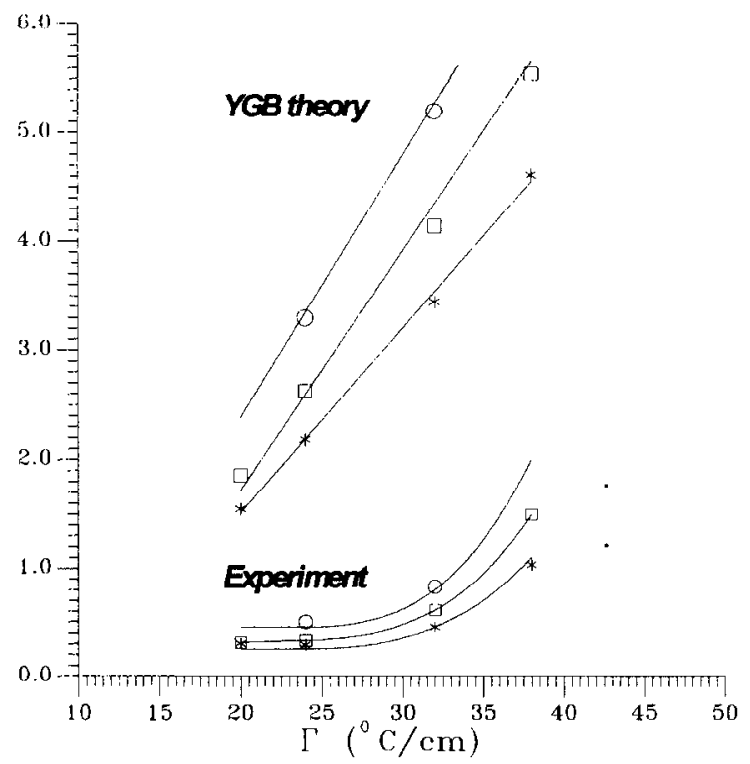

Fig. 3. The influence of temperature gradient on the thermocapillary velocity for fixed drop diameters of $8.32 \mathrm{~mm}(\mathrm{O}), 6.63 \mathrm{~mm}(\square)$ and $5.51 \mathrm{~mm}(*)$.

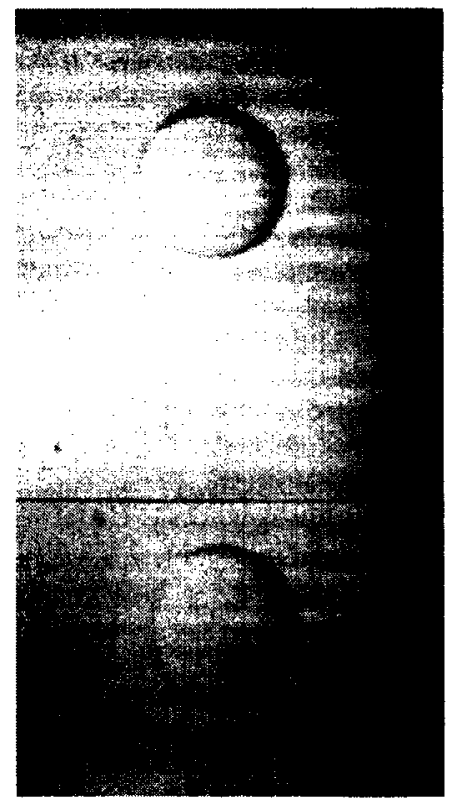

Fig. 4. Drop deformation in normal gravity (lower drop) and reinstatement in microgravity (upper drop). 


\section{DISCUSSION}

The experiment in a microgravity environment produce a drop migration driven only by the thermocapillary effect. In the present free fall experiments, the thermocapillary velocities were obtained in a microgravity environment and the features of drop migration for intermediate Reynolds number were studied. The experimental results show that the drop migrations in the case of intermediate Reynolds number of $\mathrm{O}\left(10^{1}\right)$ in magnitude have large deviation from the simplified linear model given by the YGB theory, and the drop thermocapillary velocities of the present experiments are much smaller than that suggested by the linear case. This conclusion agrees with the previous ground-based experiment (Xie et al., 1996). It implies that the non-linear effects should be considered for the drop migration for Reynolds numbers larger than 1 . When the Reynolds numbers are larger, the inertial and convective effects can not be neglected. Furthermore, non-linear effects need to be considered, and may make the drop migration feature more complex. The thermocapillary effect cannot be simply decoupled from the influence of gravity in the ground-based experiments by a non-linear model. The experimental results also indicate that the drop migration for intermediate Reynolds numbers would be submitted to viscous drag and viscous stress, which play important roles in reducing the migration velocity. That is why the migration velocity could be reduced greatly from the combined migration velocity to the thermocapillary velocity within a part of a second, when the level of gravitational acceleration changes from one-g to micro-g (see for example, Figure 1). Nevertheless, unlike the YGB model, the experimental results show that the thermocapillary velocity versus temperature gradient are not linear.

According to the experimental results, 4.5 seconds of microgravity time is not long enough for a drop approaching its steady thermocapillary velocity in the present experiment. It can also be observed that the drops had deformation when they migrated in normal gravity, due to their relatively large diameter. When the free fall began and buoyancy disappeared, the drop deformation was reinstated, see for example, Figure 4.

Preliminary experimental results are presented in this paper. More experiments at larger Reynolds numbers should be performed in a further study.

\section{ACKNOWLEDGMENT}

The project is supported partly by the National Natural Science Foundation (19789201) of China.

\section{REFERENCES}

Balasubramaniam, R., An-Ti, Chai, Thermocapillary Migration of Droplets: An Exact Solution for small Marangoni Numbers, .J. Colloid Interface Sci. 119, 531 (1987).

Balasubramaniam, R., J. E. Lavery, Numerical Simulation of Thermocapillary Bubble Migration under Microgravity for Large Reynolds and Marangoni Number, Num. Heat Transfer A 16, 175 (1989)

Braun, B., C. Ikier, H. Klein, Thermocapillary Migration of Droplets in a Binary Mixture with Miscibility Gap during Liquid, Liquid phase Separation under Reduced Gravity, J. Colloid Interface Sci. 159, 515 (1993).

Hahnel, M., V. Delitzer, H. Eckelmann, The motion of droplets in a vertical temperature gradient, Phys. Fluids, A 1, 1460 (1989).

Haj-Hariri, H., A. Nadim, and A. Borhan, Effect of Inertia on the Thermocapillary velocity of a Drop, J. Colloid Interface Sci. 140, 277 (1990).

Merritt, R. M., D. S. Morton, R. S. Subramanian, Flow Structures in Bubble Migration under the Combined Action of Buoyancy and Thermocapillarity, J. Colloid Interface Sci. 155, 200 (1993). 
Nallani, M., R. S. Subramanian, Migration of Methanol Drops in a vertical Temperature Gradient in a Silicone Oil, J. Colloid Interface Sci. 157, 24 (1993)

Subramanian, R. S., Slow migration of a gas bubble in a thermal gradient, A. I. Ch. E. J. 27, 646 (1981).

Shankar, N., R. S. Subramanian, The Stokes Motion of a Gas Bubble Due to Interfacial Tension Gradients at low to Moderate Marangoni Numbers, J. Colloid Interface Sci. 123, 512 (1988).

Wozniak, G., On the Thermocapillary Motion of Droplets under Reduced Gravity, J. Colloid Interface Sci. 141, 245 (1991).

Xie, J. C., H. Lin, J. H. Han, and W. R. Hu, Drop Migration of Middle Reynolds Number in a Vertical Temperature Gradient, Microgravity Sci. Technol. IX/2, 95 (1996).

Young, N. O., J. S. Goldstein, M. J. Block, The motion of bubbles in a vertical temperature gradient, J. Fluid Mech. 6, 350 (1959). 